\section{Richard Chole \\ Washington University in St. Louis School of Medicine}

Learning Objectives: To understand the significance of chronic, recalcitrant infections in cholesteatomas. Processes including biofilm formation and bacterial persistence.

Acquired and sometimes congenital cholesteatomas, often become chronically infected. The most common organisms associated with infected cholesteatomas are Pseudomonas aeruginosa and Staphylococcus aureus. Other gram negatives are common associated pathogens, such as Klebsiella, Proteus and E.coli. Infected cholesteatomas are more aggressive and destructive than uninfected cholesteatomas as evidenced by clinical observation and studies in experimental models of cholesteatoma.

The eradication of bacterial infections within cholesteatomas has proven difficult. Treatment with systemic and topical antibiotics often fails to eradicate the infection even though the involved organisms are sensitive to the antibiotics used. The mechanisms of bacterial resistance intolerance in cholesteatomas are complex. There are several possible mechanisms for the tolerance of chronic clinically bacteria in chronically infected cholesteatomas. These include: 1) sequestration of the cholesteatoma matrix from the general circulation; 2) ineffeective penetration of topically applied antimicrobials; 3) formation of microbial biofilms within the cholesteatoma with the resultant change in phenotype to be tolerant to host defenses and antibiotics; and 4) formation of persister cells in bacterial colonies. These cells while viable at a very low metabolic rate and low levels of replication. This change makes this persister cell type highly resistant to antimicrobials.

Strategies to eradicate biofilm infections and the presence of persister cells will be discussed.

doi:10.1017/S0022215116001547

\section{Petrous Cholesteatoma (R631)}

\section{ID: 631.1}

\section{Cholesteatoma of the Petrous Apex: Facial Nerve Management}

Presenting Author: Richard Irving

Richard Irving

Queen Elizabeth Hospital

Learning Objectives: Both congenital and acquired cholesteatomas are found in the petrous temporal bone, with the latter being more common. Congenital cholesteatomas arising in the apex erode from the medial aspect involving the facial nerve more frequently in its IAC and labyrinthine segments. Congenital middle ear cholesteatomas tend to present earlier with a conductive hearing loss and if left untreated can erode along the facial nerve superior to the cochlea involving the geniculate ganglion and labyrinthine segments. Acquired cholesteatomas involving the apex in contrast all arise in the tympanomastoid region and extend medially. These extensive acquired cholesteatomas can involve multiple segments of the intratemporal nerve from IAC to stylomastoid foramen. In the authors experience $50 \%$ of cases of apical cholesteatoma had facial nerve involvement at presentation.

The management of apical disease remains contentious. While the aim of surgery should be complete excision of disease, this should be balanced against achieving a good neurological outcome for the patient. Complete excision is often complicated by limitations in access and the tight adherence of cholesteatoma matrix to key structures such as the facial nerve, internal carotid artery and dura. The author would typically not sacrifice a functioning facial nerve in order to improve the chance of complete excision of disease. Using this approach long-term disease control and good facial motor function can be achieved in both congenital and acquired apical cholesteatoma.

doi:10.1017/S0022215116001559

\section{Petrous Cholesteatoma (R631)}

\section{ID: 631.2}

The role of the Transotic approach in cases of Petrous Bone Cholesteatoma

Presenting Author: Miguel Arístegui

Miguel Arístegui

Hospital General Universitario Gregorio Marañón Madrid Spain

Learning Objectives: We will show the benefit of the transonic approach as a safe procedure to preserve facial nerve function, to achieve total resection and to prevent complications, in cases of Petrous Bone Cholesteatoma.

Petrous Bone Cholesteatoma is a life threatening condition. Acquired or congenital in origin, is one of the most challenging intratemporal lesions.

Depply located inside the petrous portion of the temporal bone the difficulty to manage this lesions is conditioned by the involvement of structures like the otic capsule, the facial nerve, the dura, the internal auditory canal, the sigmoid sinus, the jugular bulb or the internal carotid artery.

Techniques that include subtotal petrosectomy are commonly associated to prevent future infections. Preservation of hearing is not a reasonable objective in many cases if total resection should be accomplished. The transtic approach with elimination of the anterior and posterior otic capsule leaving the facial nerve in place, adapts to some of this challenging lesions.

We report on a series of 60 Petrous Bone Cholesteatoma A Modified Sanna Classification is used to define the location and extension of the lesions.

We will report on hearing function, facial nerve function and complications.

We will focuss on those cases in which we have used the transotic approach to preserve anatomically and functionally the facial nerve. 